\title{
QUANTITATIVE 3D RECONSTRUCTIONS AS IDENTIFICATION TOOL IN HEART DEVELOPMENT
}

\author{
AleXANDre T SOUfAn, JAN M RuiJTER, MAuricE JB VAN DEN HoFF AND ANTOON FM \\ MOORMAN \\ Experimental \& Molecular Cardiology Group, Academic Medical Center, K2-283, Meibergdreef 15, 1105 AZ \\ Amsterdam, the Netherlands \\ e-mail: A.T.Soufan@amc.uva.nl \\ (Accepted August 10, 2001)
}

\begin{abstract}
A method for displaying quantitative information in 3D reconstructions of the embryonic heart was developed to investigate spatial distributions of cell division and cell density. The method utilizes serial sections to extract morphological as well as quantitative data. The morphological data are used to reconstruct the embryonic heart and the quantitative data are classified and superimposed on the resulting reconstruction. The bias, which would result from size differences between cell populations, was investigated. If present, it would influence the absolute number of particles (nuclei) per volume, although the classification applied on the reconstruction displaying the mitotic fraction remains unchanged. Although the reconstruction displaying the local densities is influenced by the bias, less than $2.5 \%$ of the regions is misclassified.
\end{abstract}

Keywords: BrdU, heart development, myocardium, quantification, 3D reconstruction.

\section{INTRODUCTION}

With embryonic development the primary linear heart tube becomes transformed into a four-chambered heart. This change in morphology involves processes like cell division, cell migration, and cell death, which result in quantitative differences in cellular organization between different parts of the heart. Most developmental studies, with 3D reconstructions, focus merely on qualitative morphological changes. Although attempts in displaying qualitative and quantitative information of heart cells in 3D reconstructions were made, interpretation of the results of these studies is hampered by the unclear definition of measured parameters and/or the confusing visualization of the results (Thompson et al., 1987; 1990). Our aim is to develop a procedure to determine and display local cell division and cell density data in their proper morphological context. The result will be a series of quantitative $3 \mathrm{D}$ reconstructions of the developing heart.

\section{METHODS AND RESULTS}

\section{STAINING}

DNA synthesizing cells of a rat embryo of 13 days of development (E13) were labelled in utero for 24 hours with bromodeoxyuridine (BrdU) prior to sacrifice. The embryo was isolated, fixed, embedded and serially sectioned $(7 \mu \mathrm{m})$. Each section was stained using a triple labelling, consisting of two immunohistochemical and one histochemical staining. The immunohistochemical stainings were used to identify myocardium and incorporated BrdU. The
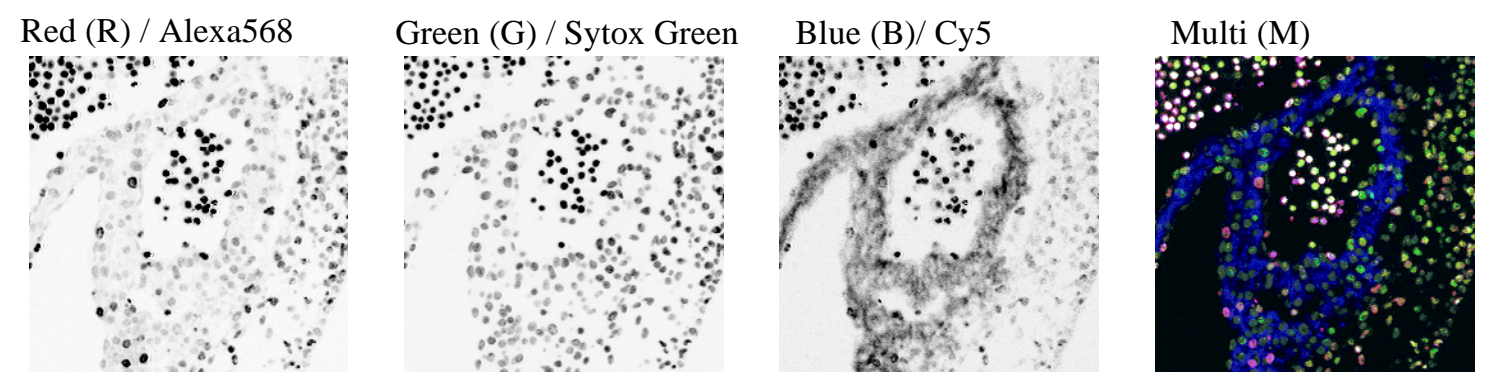

Fig. 1. Images acquired with the confocal scanning laser microscope. 
myocardium was identified using a primary antibody against myosin and visualized by a secondary antibody covalently coupled to the fluorescent dye Cy5 (Amersham Pharmacia Biotech). BrdU containing nuclei were similarly identified, using an antibody against BrdU (Becton Dickinson) and visualized using the fluorescent dye Alexa568 (Molecular Probes). For the histochemical staining of all nuclei, the fluorescent dye Sytox-Green (Molecular Probes) was used, which has a high binding-affinity for DNA.

\section{IMAGE ACQUISITION}

The stained sections were visualized - and subsequently digitised - with a confocal scanning laser microscope (CSLM), equipped with a Krypton/Argon laser (Biorad, MRC 1024). The CSLM was used for its capability of simultaneously acquiring an image from three different channels, which correspond to the emission spectra for each of the fluorescent dyes (Alexa568, Sytox-Green and Cy5). Per section a triplet of images, displaying BrdU containing nuclei, all cell nuclei or myocardium, was obtained in the red, green or blue channel, respectively (Fig. 1). The sections of the series containing myocardium (blue channel) were registered and the rotation and translation parameters obtained from this alignment were applied to the other two series (red and green channel).

\section{THRESHOLDING}

After registering, every triplet of images was processed in an image-processing program (NIHImage) using a custom written macro. The macro requiring a triplet of images as input - produced one segmented image, in which the myocardium is clearly defined and mitotic and quiescent nuclei are individually labelled. The steps implemented in the macro are schematically drawn in Fig. 2 and further elaborated below.

The first step was the removal of the profiles belonging to autofluorescent blood-cells from all three images: $\mathrm{R}, \mathrm{G}$ and $\mathrm{B}$, representing the BrdU positive nuclei, all nuclei and myocardium, respectively. To do so, these three images were added together to form an image (pooled) from which the blood cell profiles could be isolated via a threshold operation. The obtained image (blood) was used with the R, G and $\mathrm{B}$ images to remove the blood cells, resulting in the images $R^{*}, G^{*}$ and $B^{*}$.

After the removal of blood cell profiles, the remaining nuclei in the $R^{*}$ image were truly BrdU positive and are assumed to have divided. Therefore, this image was considered to contain the mitotic nuclei (mit). In order to produce the image containing the quiescent nuclei (qui), the $\mathrm{G}^{*}$ image containing all nuclei was used in a NOT operation with the $\mathrm{R}^{*}$ image. The $\mathrm{B}^{*}$ image was smoothed with erode and dilate operations and small profiles were deleted to produce a clearly defined myocardial area (myo).

The myo, mit and qui images were used in AND and OR operations (blue, red and white arrows) to obtain the mitotic myocardial cells (mit-myo) and the quiescent myocardial cells (qui-myo). The mit-myo and qui-myo images were combined via an OR operation (purple arrows) to produce an image containing both populations of myocardial cells (myo-cell).

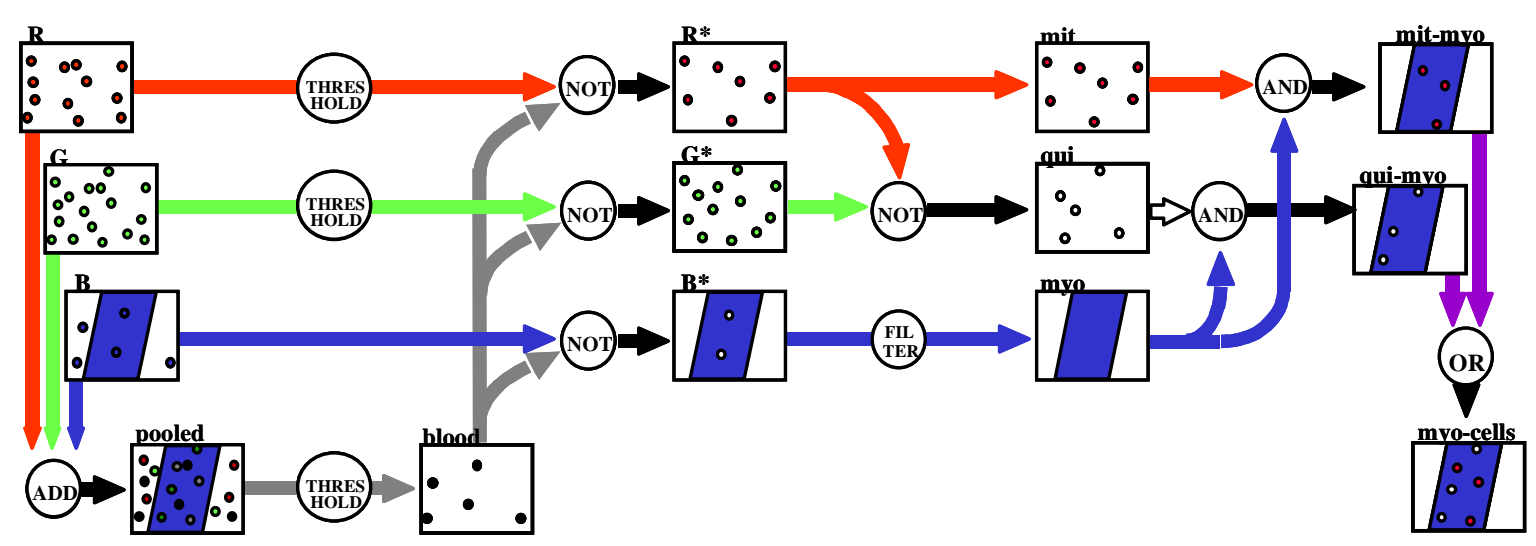

Fig. 2. Flow chart of the image processing macro. The NOT operation, should be read as e.g. R AND (NOT blood) gives $R^{*}$. 
A

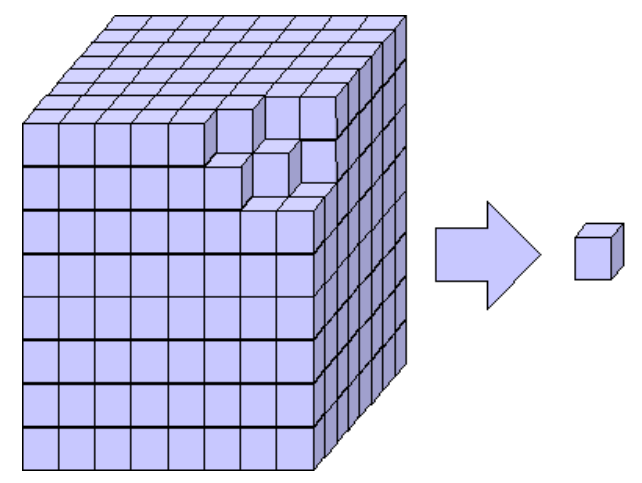

B

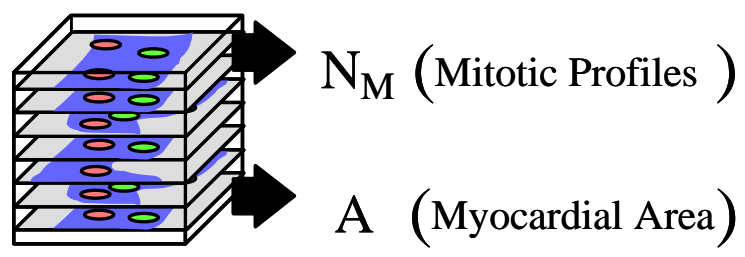

Density

(profiles per area)

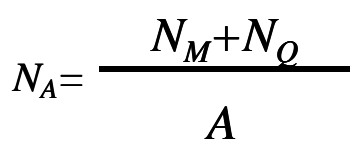

Mitotic Fraction (based on profiles)

$$
M F_{A}=\frac{N_{A M}}{N_{A M}+N_{A Q}}
$$

Fig. 3. A: The complete reference space of the virtual stack is systematically probed. B: Profiles contained within each probe are used to calculate corresponding mitotic fraction and profile density. $N_{A M}$ and $N_{A Q}$ are the number of mitotic and quiescent nuclear profiles per area, respectively.

\section{MEASUREMENT}

Next, a virtual stack was made of the myo-cell images, $7 \mu \mathrm{m}$ apart, corresponding to the original thickness of each section. The complete reference space was systematically probed using a cube of (50 $\mu \mathrm{m})^{3}$ (Fig. 3A). The number of profiles of mitotic $\left(N_{M}\right)$ and quiescent nuclei $\left(N_{Q}\right)$ as well as the myocardial area contained within each probe were measured and used to calculate the mitotic fraction (the percentage of cells undergoing division) and the density (nuclear profiles per $\mathrm{mm}^{2}$ ) within the $(50$ $\mu \mathrm{m})^{3}$ volume of interest (Fig. 3B).

\section{CLASSIFICATION}

The probing results were then used to construct frequency distributions of the mitotic fraction and the profile density. The area under the curves was divided into three classes, the bottom $25 \%$ (cyan) is designated as low, the middle $50 \%$ as medium and the top 25\% as high (red) (Fig. 4).

The corresponding parameter values were then used to subdivide the myocardial area of the sections into regions displaying low, medium, and high mitosis and density, respectively, resulting in two series of classified images (Fig. 4).

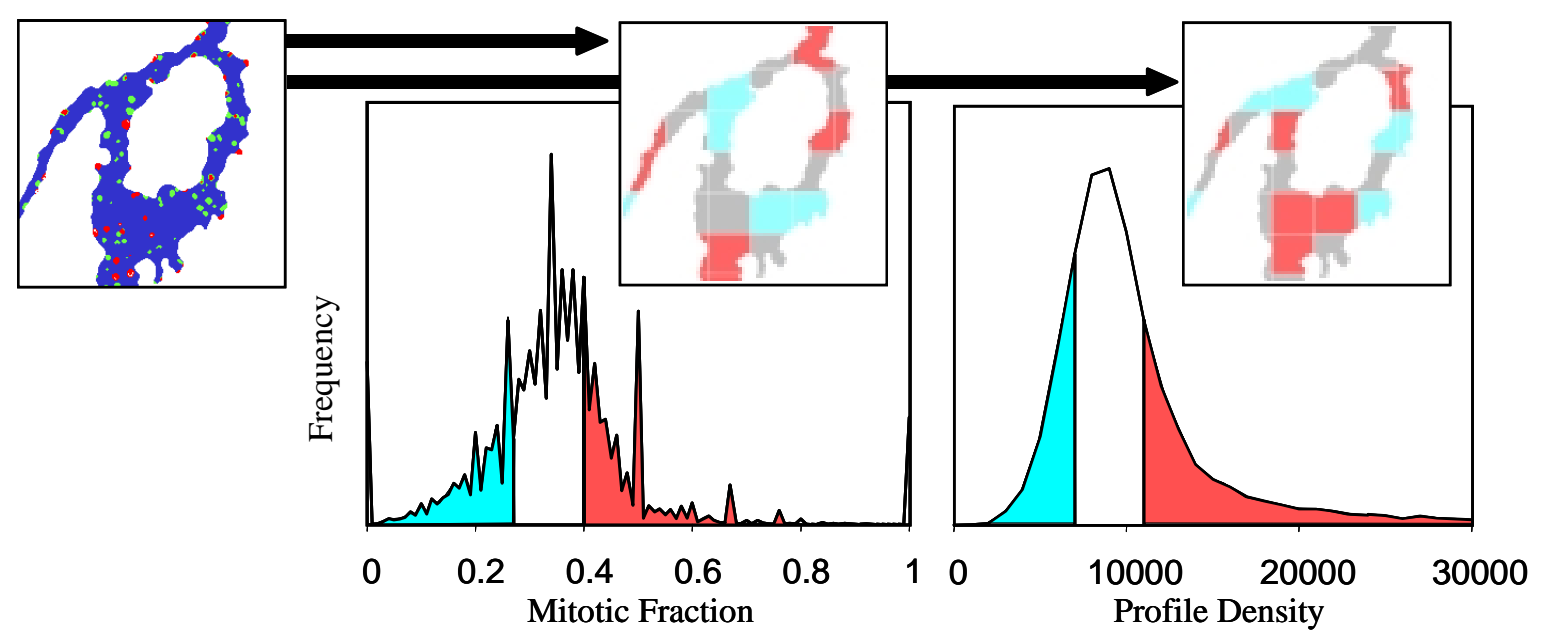

Fig. 4. The frequency distributions of the mitotic fraction and density plotted and divided into three classes. The corresponding parameter values are used to subdivide the myocardial area of the sections into regions displaying low (red), medium (grey), and high (red) mitosis and density, respectively. 


\section{RECONSTRUCTION}

Using an advanced visualization program (Amira 2.2, TGS), the two series of classified images were stacked and rendered. This resulted in two reconstructions of an embryonic heart, displaying the quantitative information on either mitosis (Fig. 5A) or cell density (Fig. 5B), both visible in three classes (low, medium and high, depicted in cyan, grey and red, respectively) in the proper morphological context.

\section{DISCUSSION}

In the method used to obtain these quantitative reconstructions, certain biological assumptions were made. It was assumed that at this developmental stage each cell contains only one nucleus, thus statements made regarding nuclei also hold true for cells and vice versa. This assumption is reasonable because multinucleation and polyploidization have been described in mouse after birth only (Soonpa et al., 1996). Secondly, the thymidine-analogue BrdU, which is incorporated into DNA during the S-phase of the cell cycle, was assumed to be a marker for mitosis.

In the present procedure, the classification of mitotic fraction and profile density (Fig. 4) is based on numbers derived from the counting of nuclear profiles (Fig. 3B). However, it would have been more appropriate to base the classification on the number of cells per volume. This would exclude the bias in the profile counts resulting from a possible difference in nuclear size of mitotic and quiescent cells. The relationship between number of profiles per area $\left(N_{A}\right)$, number of particles per volume $\left(N_{V}\right)$, nuclear diameter $(D)$ and section thickness $(t)$ is expressed in the equation of Abercrombie: $N_{V}=N_{A} /(D+t)$ (Weibel, 1979), which assumes that the nuclei are spherical or at least fully convex. Although it has been described that myocyte nuclei in adults are shaped like disks (Gerdes, 2001), this is not yet the case in E13 embryos, as all profiles seen in sections are perfect circles, indicating spherical nuclei. Because the difference in diameter of dividing and quiescent nuclei could not be measured at the magnification used, the effect of such a size difference was determined theoretically.

From the equation of the mitotic fraction based on profiles $\left(M F_{A}\right.$; Fig. $\left.3 \mathrm{~B}\right)$, the above equation of Abercrombie and the size difference $f$ between mitotic and quiescent nuclei as defined by Eq. 1

$$
f=\frac{D_{M}+t}{D_{Q}+t},
$$

the relation between the mitotic fraction based on number per volume $\left(M F_{V}\right)$ and mitotic fraction based on profiles per area $\left(M F_{A}\right)$ is given by Eq. 2

$$
M F_{V}=\frac{M F_{A}}{f-f \cdot M F_{A}+M F_{A}} .
$$

To obtain a reasonable maximum value for $f$, it can be assumed that during mitosis the nuclei would not more than double their volume compared to quiescent nuclei, increasing $D_{Q}$ by a factor $\sqrt[3]{2}$. Using a mean nuclear diameter for quiescent nuclei $\left(D_{Q}\right)$ of $10 \mu \mathrm{m}$, this results in a $D_{M}$ of $10 \cdot \sqrt[3]{2} \mu \mathrm{m}$. With these diameters and a section thickness $(t)$ of $7 \mu \mathrm{m}$, Eq. 1 results in a maximum $f$ value of approximately 1.15 .
A

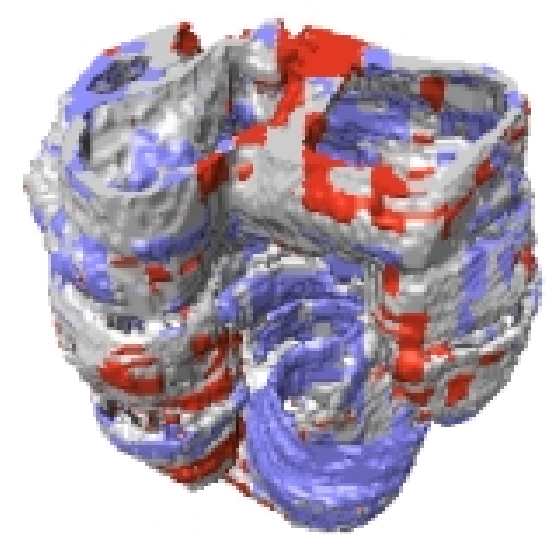

B

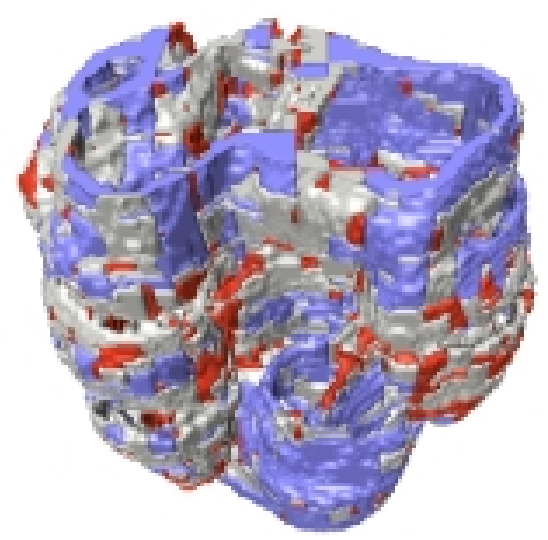

Fig. 5. Quantitative reconstructions of E13 rat heart myocardium. A. Mitotic fraction. B. Profile density. 
A plot of Eq. 2 using $f$ values of 1 and 1.15 (Fig. 6A) shows that doubling the nuclear volume has a minimal influence on the mitotic fraction. The maximum shift between the observed mitotic fraction based on profiles $\left(M F_{A}\right)$ and actual mitotic fraction based on cells $\left(M F_{V}\right)$ is $3.5 \%$ at an $M F_{V}$ of 0.53 . Moreover, the relation between $M F_{V}$ and $M F_{A}$ is a one to one transformation and, therefore, a change from profiles to particles does not influence the classification in low, medium and high mitotic activity. It can be concluded that the classification of the mitotic fraction is independent of the size difference between dividing nuclei and quiescent nuclei. Therefore, a classified mitotic fraction based on profile number per area can be interpreted as mitotic fraction per volume.

Unfortunately, it cannot be assumed that the same holds true for the cell density classes. The relation between the particle and profile densities with a size difference $f$ between quiescent and mitotic nuclei is given by the following extension of Abercrombie's equation (Eq. 3):

$$
N_{V}=\frac{N_{A Q}+N_{A M} / f}{D_{Q}+t} .
$$

From the fact that - in contrast to Eq. 2- $N_{A Q}$ and $N_{A M}$, the number of quiescent and mitotic profiles per area, respectively, are both still present in Eq. 3, it is clear that the relation is not one to one. The observed profile densities from the data set were used to plot the cell density calculated with $f=1$ (Eq. 1 ; no size difference) against cell density when the size difference is maximal $(f=1.15)$. The resulting plot (Fig. 6B) forms a narrow wedge of which the lower limit is determined by observations without mitotic cells $(y=x)$ and the upper limit by those with only mitotic cells $(y=x / f)$. Thus, when equal nuclear sizes are assumed, the number of cells per volume are overestimated $f$ times. This only happens in the sparse regions with $100 \%$ dividing cells; for the average mitotic fraction of 0.35 and a size difference $f$ of 1.15 , the deviation will be about 5\%. When the frequency distribution of cell density with this maximum size difference was divided into low, medium and high classes, the classification of only $2.5 \%$ of the observations was altered. This misclassification hardly affects the resulting $3 \mathrm{D}$ reconstruction.

Despite the uncertainty about the size difference of mitotic and quiescent cells, the cell numbers observed in this study closely resemble published data. In a study of murine myocardium, counting nuclei in $4 \mu \mathrm{m}$ thick sections of an embryonic developmental stage corresponding to the rat stage used in this study, it was found to contain 7500 nuclei $/ \mathrm{mm}^{2}$ (Cluzeaut and Maurer-Schultze, 1986). Using Abercrombie's equation, this number was used to calculate the number of nuclei per $\mathrm{mm}^{3}$ and compared to the overall number of nuclei per $\mathrm{mm}^{3}$ found in the present study. Interestingly, the resulting densities are similar: $5.4 \cdot 10^{5}$ cells $/ \mathrm{mm}^{3}$ in mouse and $4.9 \cdot 10^{5}$ in rat. Still, to obtain unbiased values of cell density and cell division a sound stereological estimator like the disector should replace the profile counting approach (Howard and Reed, 1998).
A

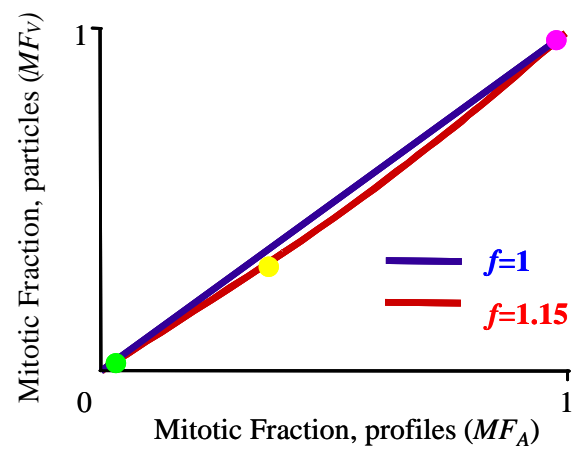

B

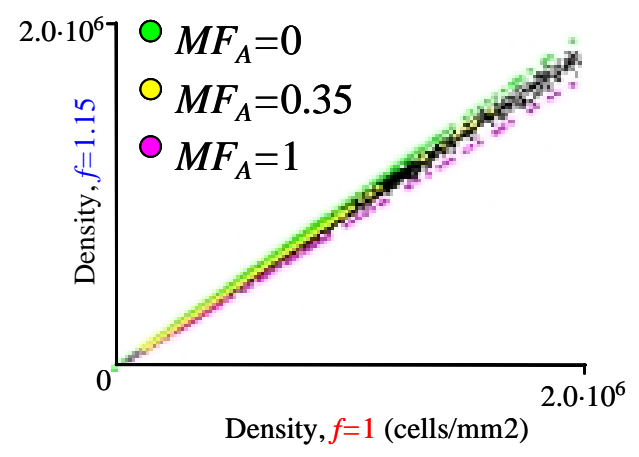

Fig. 6. The influence of a size difference between mitotic and quiescent nuclei on the quantitative data. A. Plot of mitotic fraction based on profiles against the fraction based on particles (Eq. 2), for $f=1$ and $f=1.15 . B$. Scatter plot of cell densities according to Eq. 3. $x$-axis: $f=1$ (no size difference) y-axis: $f=1.15$. 


\section{ACKNOWLEDGEMENTS}

The authors were supported by a grant from the Netherlands Heart Foundation (M96.002). They thank Jaco Hagoort and Sabine Tesink-Taekema for their support.

\section{REFERENCES}

Cluzeaut F, Maurer-Schultze B (1986). Proliferation of cardiomyocytes and interstitial cells in the cardiac muscle of the mouse during pre- and postnatal development. Cell Tissue Kinet 19:267-74.

Gerdes AM, Morales MC, Handa V, Moore JA, Alvarez MR (2001). Nuclear size and DNA content in rat cardiac myocytes during growth, maturation and aging. J Mol Cell Cardiol 23:833-9.

Howard CV, Reed MG (1998). Unbiased Stereology:
Three-dimensional measurement in microscopy. Ch. 5 Oxford: BIOS Scientific Publishing, 96-106.

Soonpaa MH, Kim KK, Pajak L, Franklin M, Field LJ (1996). Cardiomyocyte DNA synthesis and binucleation during murine development. Am J Physiol 271:H2183$\mathrm{H} 2189$.

Thompson RP, Lindroth JR, Wong YMM (1990). Regional differences in DNA-synthetic activity in the preseptation myocardium of the chick. In: Clark EB, Takao A, eds. Developmental cardiology: morphogenesis and function. Mount Kisco, NY: Futura Publishing Co., 219-34.

Thompson RP, Abercrombie V, Wong M (1987). Morphogenesis of the Truncus Arteriosus of the Chick Embryo Heart: Movements of Autoradiographic Tattoos During Septation. Anat Rec 218:434-40.

Weibel ER (1979). Stereological Methods. Vol. 1, Ch. 2. London: Academic Press, 9-62 